\title{
Déterminisme de l'eutrophisation de la retenue de Grangent (Loire) : Etude des apports en nutriments, de la dynamique des populations phytoplanctoniques et des relations phyto-zooplancton en 1990-1991
}

Impact of eutrophication in Grangent reservoir (Loire, France) : Study of the nutrient loads, the dynamics of phytoplankton populations and phyto-zooplankton relations in 1990-1991

\author{
J.L. Berthon ${ }^{(1)}$, J. Devaux(2), L. Aleya(3), H. Giraudet ${ }^{(1)}$ \\ et $F$. Restituito ${ }^{(4)}$ \\ (1) Université Jean Monnet - Laboratoire de Biologie animale et appliquée, 23 rue du Docteur \\ Paul Michelon, 42023 Saint-Etienne Cedex. \\ (2) Université Blaise Pascal, Clermont-Ferrand II. \\ (3) Université de Franche-Comté, Besançon. \\ (4) Eco-Hydro Services, 63670 Orcet.
}

Résumé. - Dans le cadre d'une approche pluridisciplinaire de l'eutrophisation de la retenue de Grangent (Loire), nous avons étudié, en 1990-91:

- les cycles biogéochimiques de divers éléments chimiques et notamment du phosphore et de l'azote;

- la physico-chimie de la colonne d'eau;

- la dynamique des populations phyto et zooplanctoniques;

- le couplage développement de Microcystis aeruginosa (M.a.)/ rapport massique N/P. Les apports externes en azote ont trois sources diffuses: le lessivage des sols couverts de végétation, les épandages d'engrais et les rejets urbains. Les apports en orthophosphates ont eux, une origine ponctuelle et urbaine. L'évolution du rapport N/P confirme cette origine: il prend des valeurs très faibles $(2,5-5)$ en fin de période d'étiage en été. Les flux d'entrée de phosphore ont été estimés à $159 \mathrm{t}$ par an $(99 \mathrm{t}$ pour la Loire, 38 pour l'Ondaine et 22 pour le Lizeron et la Semène, affluents arrivant directement dans la retenue). Le flux de sortie est de $86 \mathrm{t}$ par an. Le stockage interne est donc de $73 \mathrm{t}$ pour 1990.

S'agissant du sédiment, le rapport N/P mesuré sur le matériel particulaire oscille autour de 2,3 (faible). La matière organique (MO) produite dans la retenue ne constitue qu'une 
part minoritaire du flux de sédimentation, le phosphore est associé à des complexes minéraux.

Le rôle du sédiment dans l'enrichissement de la colonne d'eau est faible lorsque l'hypolimnion est oxygéné. Dans les eaux surnageantes du sédiment, le phosphore est sous forme organique. Dans les eaux interstitielles, le $\mathrm{P}_{-} \mathrm{PO}_{4}$ est assez abondant. C'est une source potentielle de $P$ biodisponible en cas d'anoxie. Mais de ce fait, cela ne constitue pas le facteur déterminant des blooms cyanobactériens, l'anoxie ne survenant qu'après leur développement. Toute tentative de restauration de la retenue par oxygénation de l'hypolimnion et/ou l'épandage de composés accepteurs d'électrons sur le sédiment, parait donc illusoire.

A la confluence de l'Ondaine on note une forte teneur en métaux lourds dans les sédiments, directement apportés par la rivière.

Dans la partie amont de la retenue, la colonne d'eau est très stratifiée en été. En juillet, la forte minéralisation des eaux en profondeur témoigne de la dégradation de la MO sédimentée.

Le développement des fleurs d'eau phytoplanctoniques, constituant la manifestation la plus évidente de l'eutrophisation, dépend directement des apports externes en phosphore et en azote.

Toutefois, l'apparition des blooms de M.a. résulte de la chute du rapport massique N/P en dessous du seuil exigé par les algues (soit 7). Lorsqu'il atteint des valeurs inférieures à 5 , les algues périclitent et laissent la place aux cyanobactéries qui acceptent un N/P faible. Cependant dès que ce rapport réaugmente en automne, les algues réapparaissent et $M . a$. régresse sans doute à cause du brassage des eaux et de ses médiocres qualités de compétiteur.

Lorsque M.a. se développe, le contenu énergétique de la biomasse zooplanctonique chute de façon importante. La plupart des animaux refuse cette cyanobactérie dans leur nourriture. Ils survivent alors en consommant les réserves accumulées tant que le phytoplancton était composé d'algues, pour compenser le jeûne partiel. Ceci conduit à un dysfontionnement de l'ensemble du réseau trophique et même à la disparition de certaines populations dont le maintien est lié à la constitution de réserves importantes, avant une époque précise ( $p$. ex. pour se reproduire).

Mots clés. - Retenue, eutrophisation, fleur d'eau, Azote et Phosphore, Cyanobactéries, Microcystis aeruginosa.

Abstract. - In the framework of a multidisciplinary study of the eutrophication of Grangent reservoir (Loire, France), we focused in 1990-1991 on:

- the biogeochemical balance of several chemical elements, most particularly phosphorus and nitrogen,

- the physico-chemical characteristics of the water column,

- the dynamics of phyto- and zooplankton populations,

- the coupling of development of Microcystis aeruginosa (M.a.) and the N/P ratio.

External nitrogen loads have three diffuse sources: leaching of ground covered with vegetation, chemical fertilizers and urban sewage. $\mathrm{P}_{-} \mathrm{PO}_{4}$ supplies are point loads of urban origin. The evolution in the N/P ratio confirms this observation: it reaches very low values (2.5-5) at the end of the summer low-water period.

Inflows of phosphorus were estimated at 159 tons per year (99 $t$ for the Loire, 38 for the Ondaine and 22 for the Lizeron and Semène, affluents arriving directly in the reservoir). The outflow is 86 tons per year. Internal storage thus represent 73 tons for 1990. 
The N/P ratio measured in sedimented particulate matter varies around 2.3 (low). The organic matter produced in the reservoir contributes only slightly to the sedimentation flux, and the phosphorus is associated with mineral compounds.

When the hypolimnion is oxygenated, sediment plays only a minor role in enriching the water column.

The phosphorus in overlying waters is mainly organic. In the interstitial waters, $\mathrm{P}_{-} \mathrm{PO}_{4}$ is relatively abundant, providing a potential source of bioavailable phosphorus in the case of anoxia. Because of this, however, it is not the decisive factor in Cyanobacteria blooms, as anoxia occurs only aftertheir development. Any attempt to regenerate the reservoir by oxygenating the hypolimnion or injecting electron acceptor compounds in the sediment would therefore appear to be fruitless.

At the confluence of the Ondaine, a high concentration of heavy metals is observed in the sediment, directly supplied by the river.

In the upstream part of the reservoir, the water column is highly stratified in summer. In July, the extreme mineralization of deep waters indicates the degradation of sedimented organic matter.

The development of phytoplankton blooms, the most evident sign of eutrophication, is directly dependent on external phosphorus and nitrogen loads.

The appearance of M.a. blooms is the result of the drop in the N/P ratio below the threshold needed by alga (7). When it reaches values below 5, algae collapse, yielding to Cyanobacteria which accept a low N/P ratio. As soon as the ratio rises again in autumn, however, the algae reappear and M.a. regresses, no doubt due to the mixing of the water and because M.a. is a weak competitor.

When M.a. develops, the energy content in the zooplankton biomass drops significantly. Mots animals refuse this Cyanobacteria as food. They survive by consuming the stores accumulated when the phytoplankton included algae, compensating for their partial fast. This results in a disruption of the entire trophic system and even in the disappearance of certain populations which, to survive, require the building up of large reserves before a specific period (e.g. to reproduce).

Key Words. - Reservoir, eutrophication, bloom, Nitrogen and Phosphorus, Blue-green Algae, Microcystis aeruginosa.

\section{INTRODUCTION}

La retenue de Grangent, aux portes de Saint-Etienne, sur la Loire, représente une richesse naturelle aux potentialités économiques et culturelles variées. C'est une importante réserve d'eau pour la production d'énergie et l'irrigation. De plus ce plan d'eau constitue un environnement touristique attrayant.
Cependant, l'accroissement rapide des activités humaines a provoqué au cours des dernières années une eutrophisation croissante de cet écosystème. En effet, le fleuve Loire reçoit tout au long de son cours amont, les rejets de plusieurs agglomérations et de l'agriculture, avec pour conséquences directes, un enrichissement en éléments nutritifs (azote et phosphore) entrainant une perturbation puis un dysfonctionnemennt du sys- 
tème. Celui-ci se traduit par une prolifération d'algues unicellulaires et de Cyanobactéries, dont les conséquences écologiques et économiques sont particulièrement visibles au niveau du plan d'eau.

Dès 1978 la retenue de Grangent avait été choisie par la Direction des Études et Recherches d'EDF comme site expérimental de l'étude du fonctionnement de ce type d'écosystèmes et le développement de modèles de simulation (Gaillard, 1981).

Une première approche (19781981) met en évidence le caractère eutrophe de la retenue (Garçon, 1981), avec une anoxie estivale nette et un enrichissement en ammonium $\left(\mathrm{NH}_{4}^{+}\right)$de l'hypolimnion et par ailleurs, les premières fleurs d'eau cyanobactériennes. Depuis ces phénomènes se sont largement amplifiés. La prolifération l'été de la Cyanobactérie Microcystis aeruginosa, dont le caractère toxique n'est plus à démontrer (Carmichael, 1981; Feuillade, 1992) constitue la manifestation la plus caractéristique de ce dysfonctionnement.

Une étude pluridisciplinaire a été entreprise en 1990, à la demande de l'E.P.A.L.A. ${ }^{(1)}$ de l'Agence de l'Eau Loire-Bretagne, et d'E.D.F. Ce travail a fait l'objet d'un rapport remis aux parties contractantes (Devaux, 1992) et les principaux résultats de cette étude sont repris ici, dans deux domaines :

- Les apports en nutriments, notamment azote et phosphore (sources externes et rôle des sédiments) en relation avec le débit du fleuve et l'oxygénation du fond au voisinage du sédiment.

- La dynamique des populations et le déterminisme des successions écologiques dans la communauté phytoplanctonique.

\section{MATÉRIEL ET MÉTHODES}

\subsection{Site d'étude}

Mise en eau en 1957, la retenue de Grangent (fig. 1) est située sur le cours amont de la Loire. Sa capacité est de 57,4 millions de $\mathrm{m}^{3}$, sa superficie de $365 \mathrm{ha}$, sa longueur de $21 \mathrm{~km}$ pour une largeur maximale de $400 \mathrm{~m}$. La profondeur maximale, au mur du barrage, est de $50 \mathrm{~m}$. Bien que la fonction principale de cette retenue soit la production d'électricité, le cahier des charges prévoit que le niveau

Fig. 1. - Plan de la retenue de Grangent.

(Les points 1 à 10 indiquent les carottages de sédiment. Les échantillons de plancton ont été prélevés aux points 3,4 et 9 ).

Fig. 1. - Plan of the Grangent Lake located on the upper part of the Loire River.

(The points 1 to 10 correspond to the sites of core boring. Samples of plankton were collected at the sites 3,4 and 9 ).

(1) E.P.A.L.A. = Etablissement Public pour l'Aménagement de la Loire et de ses Affluents. 


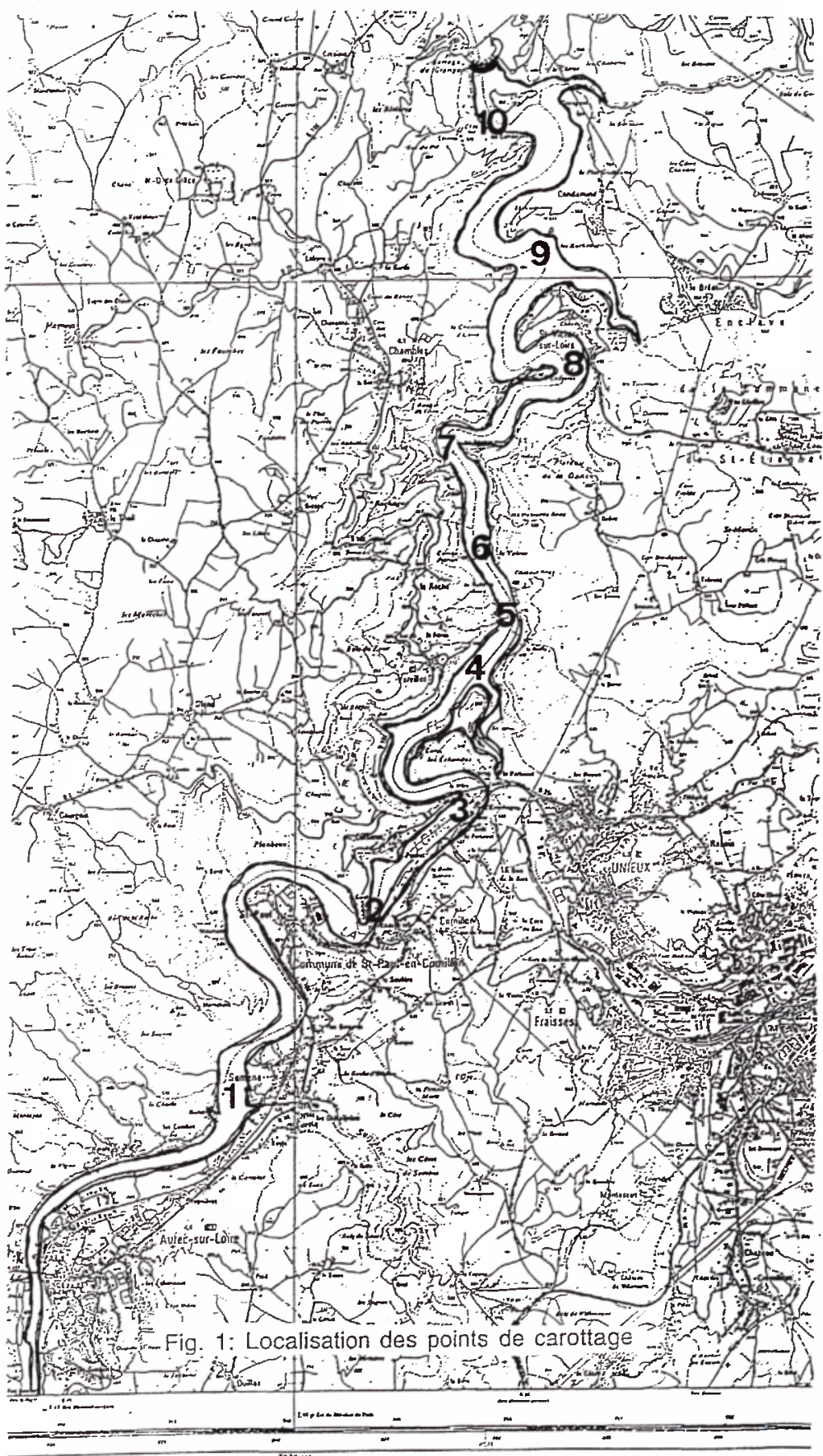


du plan d'eau doit être maintenu à la cote 420 (NGF) du $1^{\text {er }}$ juin au 15 septembre, pour l'usage touristique. La dernière vidange de l'ouvrage remonte à 1977, ce qui suggère une importante accumulation de sédiments dans la retenue.

\section{2 Échantillonnage}

Le programme de mesures et d'échantillonnages, d'une durée d'un cycle biologique, a été réalisé au cours de l'année 1990.

\section{- Localisation des points d'étude :}

La position des points de mesure est donnée sur la figure 1.

\section{- Apports externes}

Deux sites (la Loire à l'aval d'Aurec et l'Ondaine au dessus de la prise d'eau de la Noirie) ont été équipés de préleveurs automatiques, dont le pas d'échantillonnage est de 2 heures. Ces appareils ont été relevés 2 fois par semaine, mais les résultats sont donnés sous la forme de moyennes hebdomadaires pour chacun des éléments analysés (Devaux, 1992).

\section{- Apports internes}

Les échantillons ont été prélevés au cours d'une campagne de carottage, le 11-04-1990 en 10 points de la retenue répartis entre le confluent de la Semène et le barrage (fig. 1). Ces échantillons ont été analysés en suivant le protocole de Golterman et al. (1978). Ont été dosés :
- sur la phase aqueuse, les ions ammonium, nitrites, nitrates, orthophosphates, et phosphore total, la silice, le fer et le manganèse, le calcium et l'aluminium;

- sur la phase particulaire, les fractions minérales et organiques, l'azote et le phosphore totaux. Ont également été recherchés les dérivés des extractions de houille.

Le matériel particulaire en voie de sédimentation a été recueilli dans des trappes à sédiment, disposées aux points $n^{\circ} 3$ (Pertuiset), 4 (Ondaine) et 9 (St-Victor) à trois profondeurs $(-1,5 \mathrm{~m}$; milieu de la colonne d'eau et à $1 \mathrm{~m}$ du fond). Trois campagnes ont été réalisées : 13-30 août 1990; 27 novembre-27 décembre 1990 et 19 mars- 9 avril 1991. Sur le matériel récolté, on a mesuré :

- le flux de sédimentation, exprimé en poids de matière sèche par $\mathrm{m}^{2}$ et par jour;

- l'importance relative des fractions organique et minérale.

Enfin, le flux de relargage par le sédiment est mesuré à l'aide de chambres benthiques. Deux campagnes ont été réalisées entre les 26-04 et 11-05 puis entre les 13 et 30-08-90, en trois points (Pertuiset, Ondaine et Saint-Victor). Les teneurs en chlorophylle a ont été estimées selon SCOR-UNESCO (1966), sur les prélèvements bihebdomadaires.

\section{- Etude des colonnes d'eau}

Les principaux paramètres physicochimiques ont été analysés sur des prélévements d'eau collectés à l'aide 
d'une bouteille fermante de type Van Dorn en 18 campagnes, du 06-06-90 au 21-04-91. Par ailleurs des profils thermiques et d'oxygène dissous ont été établis aux trois points de récolte du plancton: Pertuiset, Ondaine et Saint-Victor (3, 4 et 9 ).

Sont mesurés: turbidité, température, $\mathrm{pH}$, oxygène dissous, conductivité, matières en suspension (MES), phosphore total (PT), orthophosphates, ammonium, nitrites, nitrates, azote total (NT) et organique (NTK), silice, sulfates, fer, manganèse et DBO5.

\section{- Phytoplancton}

Entre le 06-06-90 et le 15-03-91, 14 séries de prélèvements de phytoplancton ont été réalisées aux stations 3,4 et 9 .

Sur les échantillons les espèces ont été identifiées et les organismes dénombrés, au microscope inversé (Wild M40) et selon la méthode d'Utermöhl (1958), modifiée par Legendre et Watt (1971-72). Les biomasses spécifiques ont été calculées par la méthode de Lohman (1908).

Par ailleurs, le dosage des pigments est effectué selon les méthodes SCOR-UNESCO (1966) et de Lorenzen (1967).

\section{- Zooplancton}

Des échantillons prélevés par des traits verticaux d'un filet Juday de $88 \mu \mathrm{m}$ de vide de maille, les mêmes jours et aux mêmes stations que le phytoplancton, ont permis l'inventaire des espèces zooplanctoniques, le dénombrement des organismes par éco- phases et le calcul des biomasses spécifiques.

Pour chaque prélèvement, la composition biochimique globale (glucides, lipides, protides, chitine et cendres) et le contenu énergétique ont été déterminés (le détail des méthodes est exposé in Berthon 1988). Les valeurs énergétiques sont calculées à partir des équivalences proposées par Juday (1940) ou Brody (1945), soit 17150,39500 et 23620 joules. $g^{-1}$ respectivement pour les glucides, lipides et protides.

\subsection{Analyse mathématique}

Une analyse en composantes principales a été effectuée, après application d'une transformation $\log (x+1)$ destinée à homogénéiser les variances (Frontier, 1973). Le logiciel utilisé est celui de Doledec et Chessel (1992).

\section{RÉSULTATS ET DISCUSSION}

Les résultats complets de cette étude sont rapportés par Devaux (1992) et intégrés dans l'analyse plus globale de l'évolution du phénomène réalisée par Salençon (1995). Nous ne reprendrons ici que ceux jugés essentiels pour tenter d'expliquer le phénomène d'eutrophisation de cette retenue (fig. 2 et 3 ).

\subsection{Les débits}

L'année 1990 est relativement sèche. Le débit moyen de la Loire n'est que de $17,8 \mathrm{~m}^{3} \cdot \mathrm{s}^{-1}$. L'étiage est 

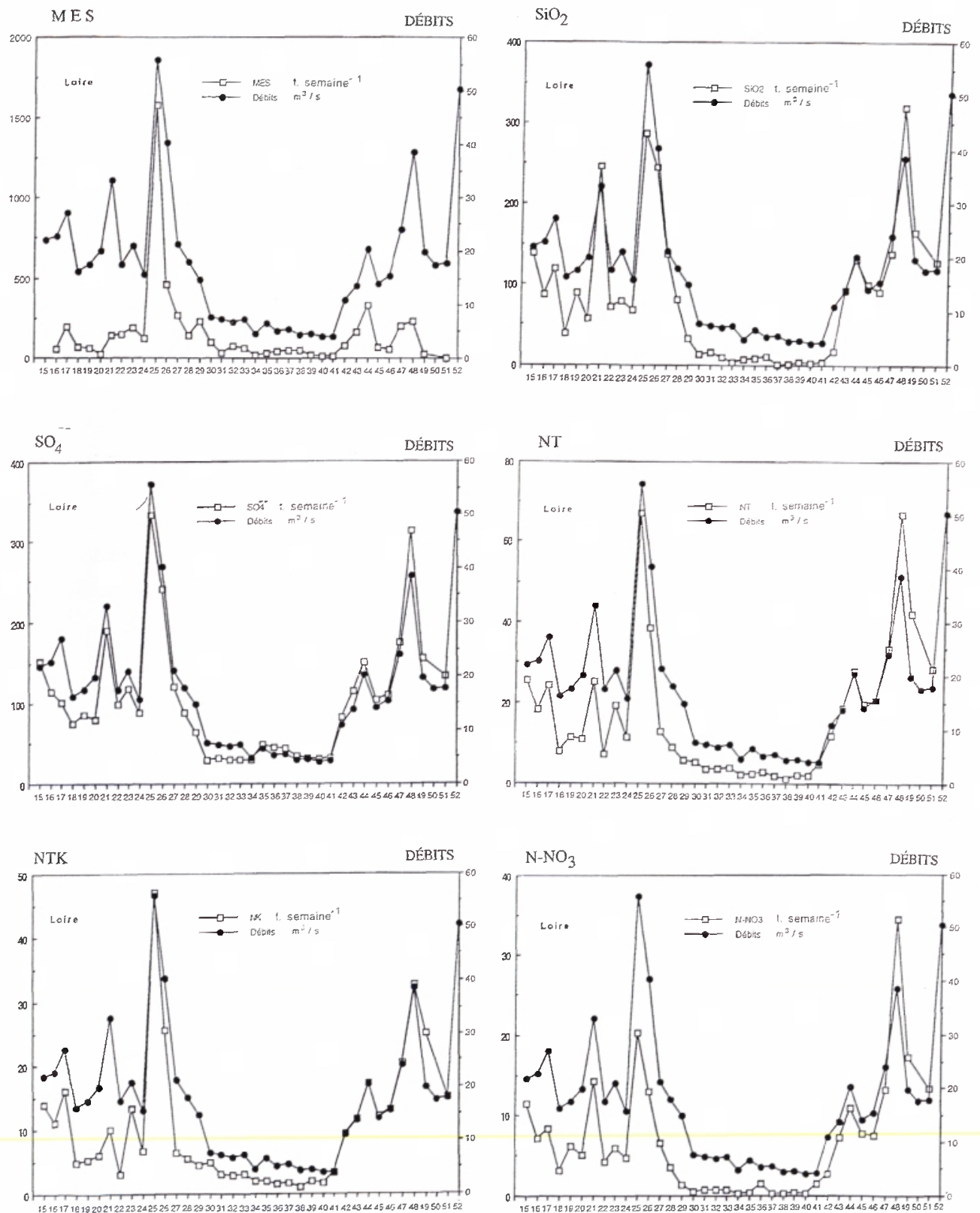

Fig. 2. - Loire - Evolution des débits, des teneurs en $\mathrm{MES}, \mathrm{SiO}_{2}, \mathrm{SO}_{4}^{--}$, Azote total (NT), Azote organique (NTK), Nitrates (N-NO 3 ), du 8-4 (Semaine 15) au 31-12-1990 (Semaine 52).

Fig. 2. - Loire River - Variations of flow, suspended matter (SM), $\mathrm{SiO}_{2}, \mathrm{SO}_{4}$, total nitrogen (NT) organic nitrogen (NTK) and $\mathrm{N}^{-N_{3}}$, from 8-4 (Week 15) to 31-12-1990 (Week 52). 

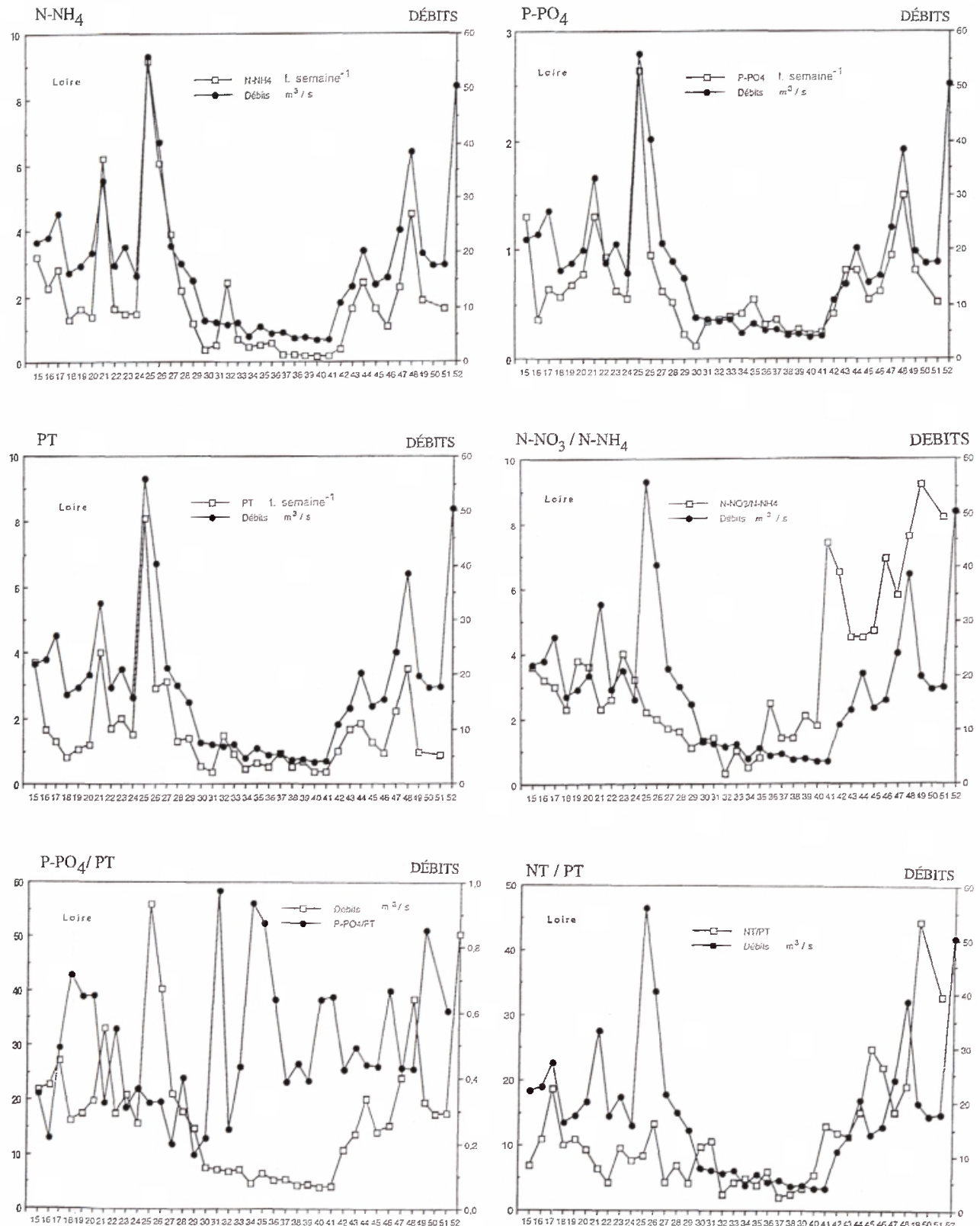

Fig. 2 (suite). - Loire - Evolution des débits, des teneurs en $\mathrm{N}-\mathrm{NH}_{4}, \mathrm{P}_{-} \mathrm{PO}_{4}$, Phosphore total (PT) et des rapports $\mathrm{N}-\mathrm{NO}_{3} / \mathrm{N}-\mathrm{NH}_{4}, \mathrm{P}-\mathrm{PO}_{4} / \mathrm{PT}$, NT/PT du 8-4 (semaine 15) au 31.12.1990 (semaine 52). Fig. 2 (next). - Loire River - Variations of flow, N-NH , $_{4} \mathrm{PO}_{4}$, total phosphorus (PT) and $\mathrm{N}$ $\mathrm{NO}_{3} / \mathrm{N}-\mathrm{NH}_{4}, \mathrm{P}-\mathrm{PO}_{4} / \mathrm{PT}$, NT/PT ratios from 8-4 (week 15) to 31.12 .1990 (week 52). 

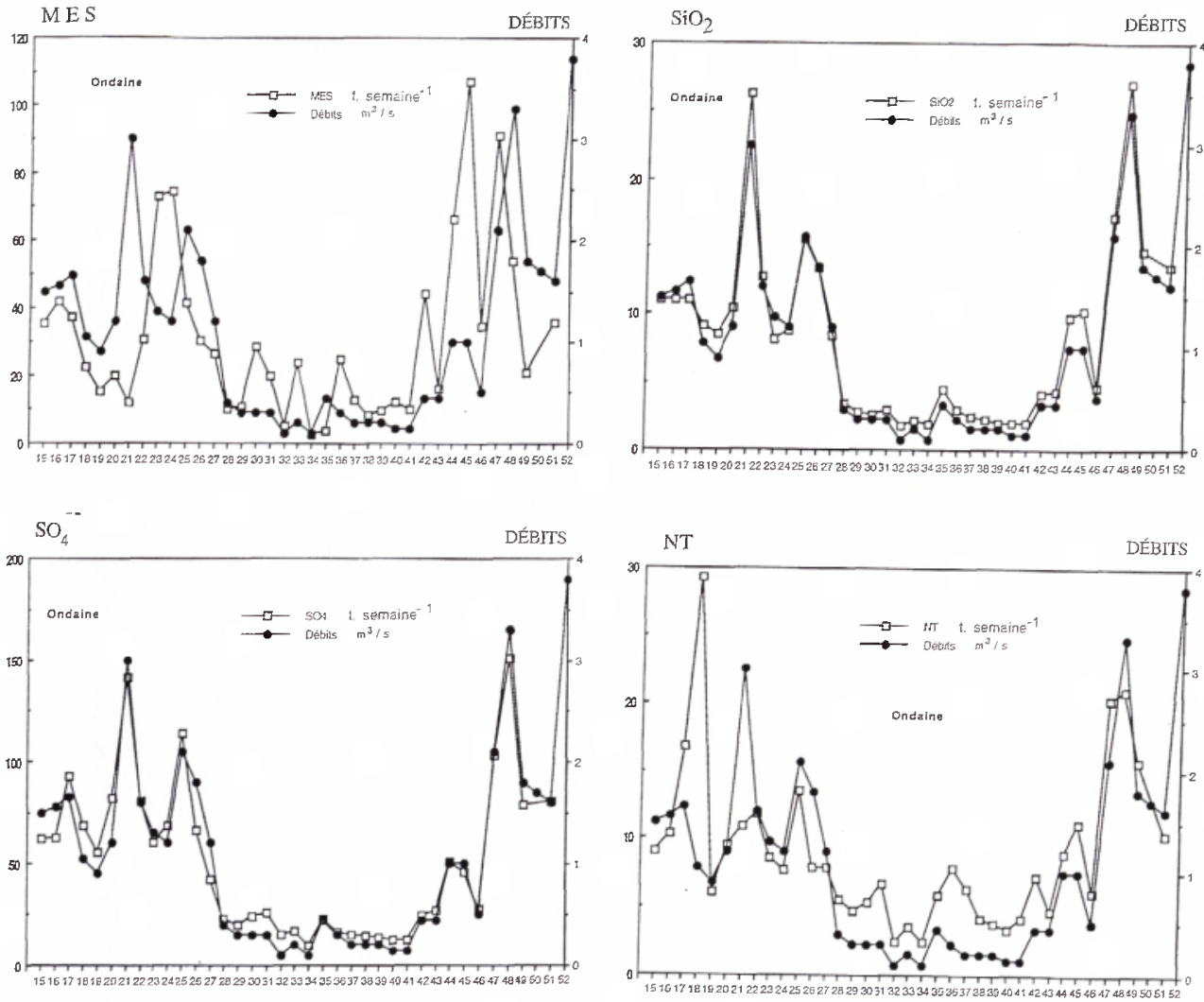

$\mathrm{N}-\mathrm{NO}_{3}$ DÉBITS $\mathrm{N}-\mathrm{NH}_{4}$ DÉBITS
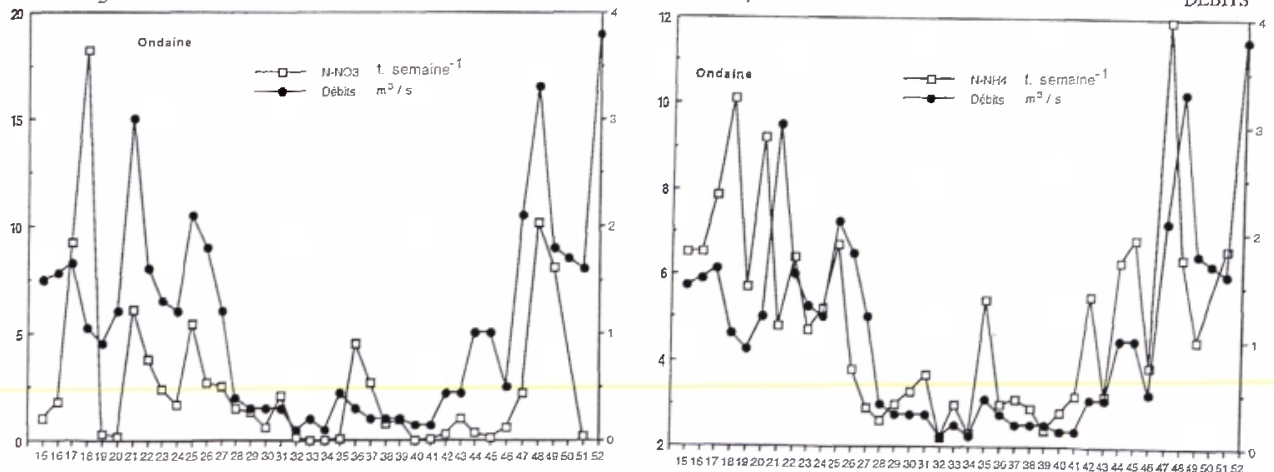

Fig. 3. - Ondaine - Evolution des débits, des teneurs en $\mathrm{MES}, \mathrm{SiO}_{2}, \mathrm{SO}_{4}^{-}$, NT, N-NO 3 et $\mathrm{N}-\mathrm{NH}_{4}$ du 8-4 (Semaine 15) au 31-12-1990 (Semaine 52).

Fig. 3. - Ondaine river - Variations of flow, suspended matter (SM), $\mathrm{SiO}_{2}, \mathrm{SO}_{\overline{4}}, \mathrm{NT}, \mathrm{N}-\mathrm{NO}_{3}$ et $\mathrm{N}-\mathrm{NH}_{4}$, from 8-4 (Week 15) to 31-12-1990 (Week 52). 

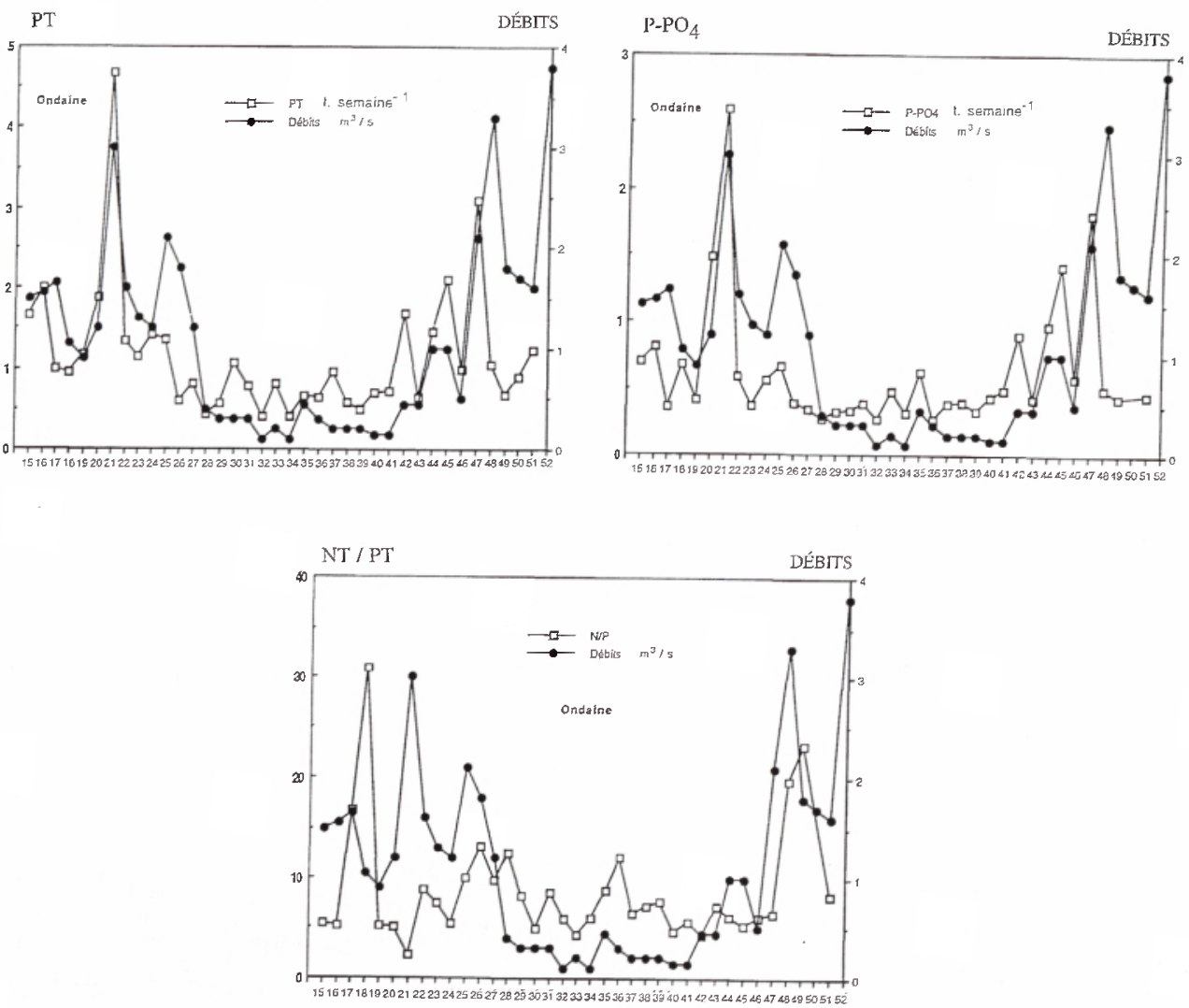

Fig. 3 (suite). - Ondaine - Evolution des débits, des teneurs en Phosphore Total (PT) P-PO du rapport NT/PT du 8-4 (semaine 15) au 31-12-1990 (semaine 52).

Fig. 3 (next). - Ondaine River - Variations of flow, total phosphorus (PT) $\mathrm{P}_{-} \mathrm{PO}_{4}$ and NT/PT ratio from 8-4 (week 15) to 31-12-90 (week 52).

inférieur à $5 \mathrm{~m}^{3} \cdot \mathrm{s}^{-1}$, tandis que la crue la plus importante ne dépasse pas $175 \mathrm{~m}^{3} \cdot \mathrm{s}^{-1}$.

Pour l'Ondaine, le débit moyen n'est que de $1,15 \mathrm{~m}^{3} \cdot \mathrm{s}^{-1}$. Tant que le débit est inférieur à $1,2 \mathrm{~m}^{3} \cdot \mathrm{s}^{-1}$, l'eau est conduite à la station d'épuration de La Noirie (60\% du temps). Au-delà elle est by-passée vers le plan d'eau. Si l'on tient compte d'une seconde dérivation lorsque la station d'épuration est elle-même saturée, l'eau de l'Ondaine, égout de l'ensemble d'une vallée très urbanisée, est rejetée directement à la Loire sans traitement, un peu plus d'un jour sur deux en 1990.

\subsection{Les apports}

\subsubsection{Les apports externes (ta- bleau 1)}

Globalement, ces apports sont corrélés avec les débits. Ceci est particulièrement vrai pour les nutriments : $\mathrm{Si}, \mathrm{N}$ et $\mathrm{P}$ sous toutes leurs formes. 
Tableau 1. - Apports annuels par la Loire et l'Ondaine au cours de l'année 1990.

\begin{tabular}{|c|r|r|}
\hline Paramètres & Loire $\left({\left.\mathrm{kg} . \mathrm{j}^{-1}\right)}^{-1}\right.$ & $\begin{array}{c}\text { Ondaine } \\
\left(\mathrm{kg.j}^{-1}\right)\end{array}$ \\
\hline $\mathrm{MES}$ & 25540 & 4300 \\
\hline $\mathrm{SiO}_{2}$ & 12000 & 1170 \\
\hline $\mathrm{N}-\mathrm{NH}_{4}^{+}$ & 280 & 700 \\
\hline $\mathrm{N}-\mathrm{NO}_{3}^{-}$ & 880 & 370 \\
\hline $\mathrm{NT}$ & 2500 & 1200 \\
\hline $\mathrm{NTK}$ & & 860 \\
\hline $\mathrm{PT}$ & 270 & 160 \\
\hline $\mathrm{P}-\mathrm{PO}_{4}^{3-}$ & 95 & 90 \\
\hline $\mathrm{SO}_{4}^{2-}$ & 14700 & 7250 \\
\hline $\mathrm{Fe}^{++}$ & 560 & 130 \\
\hline $\mathrm{Mn}^{++}$ & 48 & 25 \\
\hline
\end{tabular}

\section{- Silice}

Le lessivage du substrat cristallin est la seule source externe (Loire et Ondaine). Les pics sont fortement corrélés aux débits et donc à la pluviométrie du bassin versant. On verra plus loin que les pics de développement des diatomées le sont également.

\section{- Azote}

Les apports d'azote par la Loire suggèrent un rôle déterminant du lessivage des sols lors des épisodes pluvieux. Cependant si l'on considère le rapport azote organique/ azote total, cette interprétation doit être modulée. Le rapport NTK/NT est maximum à l'étiage, or NTK ne peut être apporté par lessivage en absence de précipitations, il est donc soit lié à la biomasse algale, soit d'origine anthropique. On retiendra la seconde origine puisque les rejets ponctuels sont dominants en phase d'étiage, ce que confirme le rapport Nitrates / ammonium.

En résumé l'azote entrant par la Loire a trois sources, d'importance variable au cours de l'année :

- le lessivage des sols couverts de végétation, dominant au printemps;

- les rejets anthropiques prépondérants en phase d'étiage;

- les apports «agricoles » par lessivage des engrais sur les sols nus, importants en automne.

Les apports en azote par l'Ondaine sont importants. Ils ne sont pas corrélés au débit. Une large part sédimente dans le lit du cours d'eau, puis est entrainée dans la retenue par les crues. Ce sont des rejets ponctuels, soit d'origine industrielle (les nitrates témoignent ici de rejets directs d'acide nitrique) soit domestique comme pour l'ammonium, dont la teneur est particulièrement élevée.

\section{- Phosphore}

Les proportions en $\mathrm{P}$ total et en orthophosphates varient de façon importante au cours de l'année, en Loire. Le rapport $\mathrm{P}_{-} \mathrm{PO}_{4} / \mathrm{PT}$ est corrélé négativement avec le débit. Le $P$ minéral, apporté par le lessivage des sols est toujours très faible, on peut dire que les apports en $\mathrm{P}_{-} \mathrm{PO}_{4}$ sont ponctuels et plutôt d'origine anthropique.

S'agissant de l'Ondaine, comme pour l'azote, les flux de phosphore total ne sont pas corrélés au débit. En 
automne et en hiver, le décalage temporel entre les pics de débits et les apports phosphorés indique une reprise de matériel sédimenté.

\section{- Rapport N/P}

Dans la Loire l'évolution du rapport massique N/P confirme l'origine urbaine du phosphore: N/P est élevé au printemps (8 à 10), lorsqu'un débit suffisant de la Loire apporte de l'azote. A l'étiage; les apports diffus d'azote diminuent mais pas ceux de phosphore (urbains). N/P chute considérablement pour atteindre 5 à 2,5 en fin d'été. Après les pluies d'automne, $\mathrm{N} / \mathrm{P}$ remonte et même dépasse 20 , valeur qui caractérise des eaux de lessivage des sols agricoles (Wetzel, 1983).

Du fait de l'importance des rejets azotés dans l'Ondaine, le rapport N/P y est toujours élevé.

\section{- Les autres apports}

Outre les apports de la Loire et de l'Ondaine, Grangent reçoit ceux de la Semène, à l'aval d'Aurec (100 kg.j ${ }^{-1}$ d'azote et $20 \mathrm{~kg}_{\mathrm{j}}{ }^{-1}$ de phosphore) et surtout ceux du Lizeron (ou Izeron) où se déversent les rejets des communes de Roche-la-Molière et de Saint-Victor. Les rejets de Saint-Victor sont bien maitrisés par une station d'épuration disposant d'un système de déphosphatation efficace, mais la station de Roche-la-Molière est largement sous dimensionnée. Elle déverse $150 \mathrm{~kg} \cdot \mathrm{j}^{-1}$ d'azote et $40 \mathrm{~kg} . \mathrm{j}^{-1} \mathrm{de}$ phosphore.

\subsubsection{Les apports internes}

\section{- Flux de sédimentation}

L'analyse du matériel particulaire récolté dans les trappes permet d'évaluer l'importance de la sédimentation et la composition de la matière sédimentée. Elle est maximale en toutes saisons au point Ondaine et minimale à Saint-Victor. Cependant il existe des différences sensibles selon les éléments considérés et selon la saison. C'est en mars-avril que le taux de sédimentation est le plus important. Outre les particules inorganiques véhiculées par les affluents et remises en suspension par les mouvements d'eau, une partie importante de la matière organique, d'origine interne, correspond à la sédimentation des diatomées, algues dominantes à cette période. Ces algues sédimentent pour une large part en parfait état physiologique et sont peu dégradées dans la colonne d'eau, comme l'indique la teneur élevée en chlorophylle $(0,2 \%$ de la M.O. contre $0,04 \%$ en été). Le rapport N/P mesuré sur le matériel particulaire oscille autour de 2,3-valeur assez faible indiquant que la matière organique formée dans la retenue ne constitue qu'une fraction minoritaire du matériel sédimentaire. Une part du phosphore est associée à des complexes minéraux.

On constate que le matériel particulaire collecté dans les trappes est bien celui qui constitue le sédiment, car sa composition chimique est identique à celle du sédiment prélevé par carottage. Quantitativement, les me- 
sures de sédimentation confirment les estimations de Coyne et Belier (1967) ou L.C.M.F. (1982) in Saunier Eau et Environnement (1988) soit entre 40 et 50000 tonnes par an. Un dépôt de $50000 \mathrm{t}^{\mathrm{a}} \mathrm{an}^{-1}$ correspond à un flux moyen de $140{\mathrm{t} . \mathrm{j}^{-1}}^{-1}$ soit $38 \mathrm{~g} . \mathrm{m}^{2} . \mathrm{j}^{-1}$, valeur très voisine de celles mesurées lors de la présente étude (Devaux, 1,992).

\section{- Teneur et relargage des nutri- ments par les sédiments}

Deux paramètres sont prépondérants quant aux potentiels de relargage de nutriments par les sédjments: l'oxydo-réduction - le phosphore n'étant dissociable des complexes métalliques qu'en conditions d'anoxie - et les stocks disponibles.

Au printemps 1990, les eaux sont encore bien oxygénées et les teneurs en orthophosphates sont faibles, notamment dans l'Ondaine. Ceci est dû aux concentrations importantes en fer et en manganèse. Un potentiel d'oxydo-réduction suffisamment élevé maintient le fer sous forme oxydée qui complexe le phosphore, donnant des composés insolubles.

En tous les points, les concentrations en $P$ total sont importantes et associées à la fraction organique.

Dans les eaux interstitielles, les concentrations en nitrates sont plus élevées que dans les eaux surnageantes. Les valeurs s'abaissent d'Aurec jusqu'au Pertuiset, puis remontent au niveau de l'Ondaine (rejets industriels d'acide nitrique et dysfonctionnement de la station d'épuration). Les teneurs en orthophosphates, fortes à l'amont, diminuent après le confluent de l'Ondaine, en relation avec l'augmentation des teneurs en fer et en manganèse. Enfin, dans la phase particulaire, les teneurs maximales d'azote s'observent à l'aval du Bec d'Ondaine. Quant au phosphore, il croit de l'amont vers l'aval. Aux stations 4 et 9 , il est essentiellement sous la forme inorganique non apatitique (lié au fer). En situation d'anoxie du fond, ce phosphore peut être solubilisé et donc être potentiellement biodisponible.

Le flux de relargage moyen, mesuré en chambres benthiques, est d'environ $35 \mathrm{mg} \cdot \mathrm{m}^{-2}$ par jour. Sur l'ensemble de la période d'étude, la quantité de phosphore relarguée serait de $5,6 \mathrm{t}$. Dans le même temps, la Loire et l'Ondaine apportent environ 23,6 t. Durant l'été, les apports internes ne sont donc pas négligeables (25\% des externes). Cependant le flux n'atteint pas la zone trophogène du plan d'eau. II ne participe donc pas, ou peu à la production algale, même dans la zone du Pertuiset, peu profonde, mais où toute la colonne d'eau demeure oxygénée tout au long de l'année.

\subsubsection{Bilan sur les apports et les sédiments}

En comparant les flux d'entrée à ceux de sortie, on peut dresser le bilan annuel pour la retenue.

Pour les MES, le flux d'entrée est d'environ $11.10^{3}$ tonnes par an, le flux de sortie n'est que de 900 tonnes. C'est donc environ 10000 tonnes de 
matériel particulaire directement apportées par les tributaires qui se sont accumulées dans la retenue durant l'année 1990. Ainsi, il apparait que la fraction la plus importante du matériel se déposant sur le fond, a une origine interne à la retenue.

Ne disposant pas du flux de sortie de l'azote total, on ne fera le bilan que des formes minérales, assimilables par le phytoplancton, de l'azote $\left(\mathrm{N}-\mathrm{NH}_{4}^{+}\right.$et $\mathrm{N}-\mathrm{NO}_{3}^{-}$). La somme des apports par la Loire, l'Ondaine et le Lizeron est de 913 tonnes par an. Or, à l'aval passent 1200 tonnes par an. II apparait donc un déficit entrée-sortie d'environ 290 tonnes par an. Même si l'on ajoute les apports supplémentaires par lessivage des rives du lac, apports qui ne peuvent être conséquents et une minéralisation d'une part de la matière organique apportée par les tributaires dans la retenue, ce calcul indique que la retenue ne stocke pas (ou peu) d'azote minéral.

Pour le phosphore enfín, les flux d'entrée sont de 160 tonnes par an. Le flux de sortie, bien corrélé au débit, est estimé à 86 tonnes par an. On aurait donc un stockage interne de 74 tonnes pour l'année 1990.

Une analyse statistique prenant en compte l'ensemble des données collectées, permet de mettre en évidence une zonation longitudinale dans la retenue, après pondération de chacun des descripteurs par l'indice de similarité de Gower (1971). Les résultats obtenus sont synthétisés par un dendogramme réalisé par un groupement agglomératif à liens complets (Devaux, 1992).
On identifie globalement 4 grandes zones:

- La zone amont (stations 1, 2, 3) où la composition chimique des eaux profondes et du sédiment est sous l'influence exclusive de la Loire.

- Une zone intermédiaire allant du confluent de l'Ondaine jusqu'aux Révottes (stations 4, 5, 6 et 7) sous la dépendance des apports de cet affluent.

- La station 8 (le Chatelet) recevant probablement des apports dont l'origine est mal connue (ancien tunnel SNCF?).

- Enfin, la zone aval, allant de Saint-Victor au barrage et dépendant des apports du Lizeron.

Dans l'ensemble, d'un point de vue quantitatif, l'important flux de phosphore parait à l'origine du développement algal. II faut rappeler que, parmi les éléments entrant dans la biomasse végétale, le phosphore est le seul n'ayant pas de source atmosphérique. II n'entre dans les écosystèmes aquatiques que par le biais des rejets liés aux activités humaines. C'est ici, l'élément déterminant du développement des fleurs d'eau. Ce rôle connu depuis longtemps a été décrit dans un modèle mathématique par Vollenweider (1982). Appliqué à Grangent, en considérant que le flux hydrique annuel est de $6.108 \mathrm{~m}^{3}$, et celui de phosphore de 160 t.an $^{-1}$ (soit une concentration en $P$ de $265 \mathrm{mg} \cdot \mathrm{m}^{-3}$ ) et que le temps de séjour des eaux est de 35 jours, ce modèle prévoit une concentration en chlorophylle a dans le lac, de l'ordre de $29 \mathrm{mg} \cdot \mathrm{m}^{-3}$. Or, 


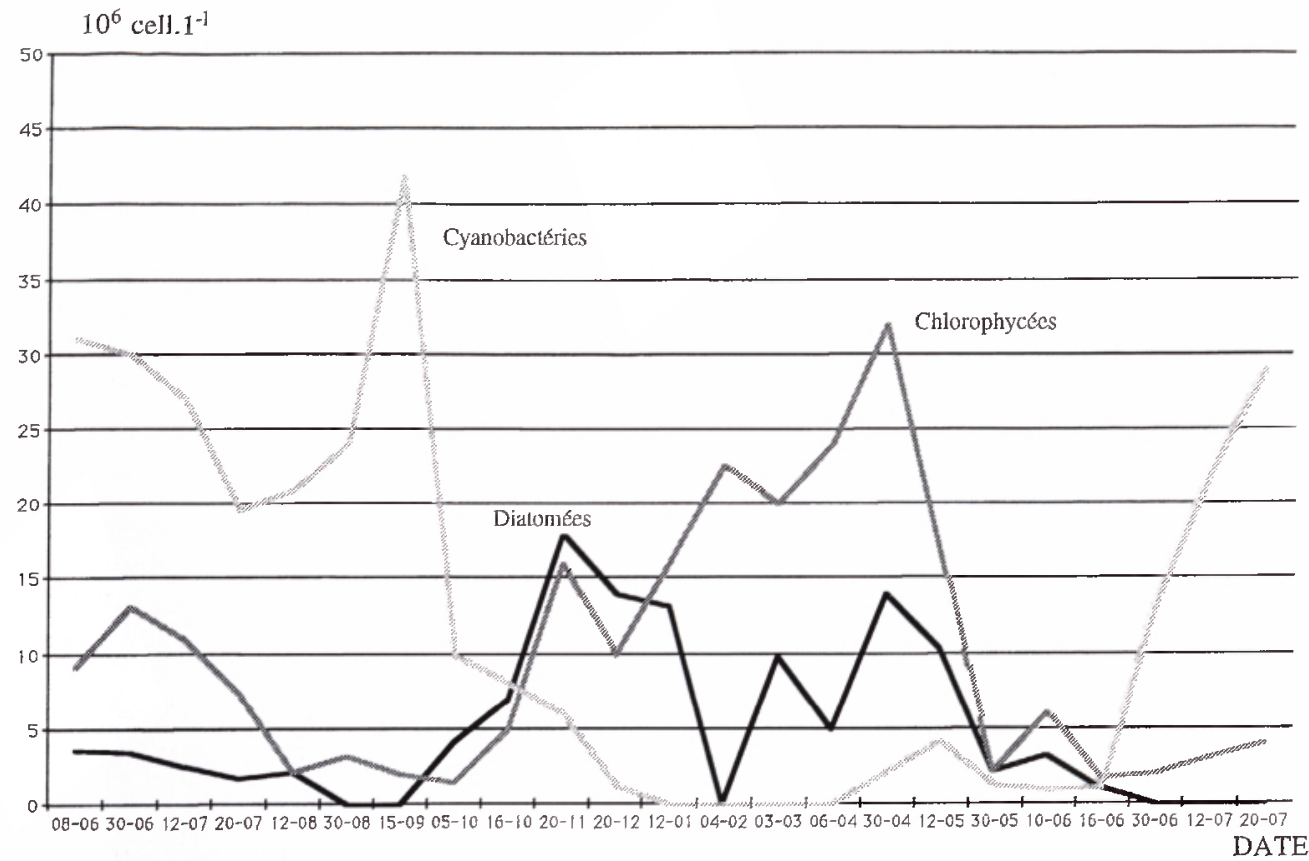

Fig. 4. - Evolution de la densité numérique (en $10^{6}$ cellules par litre) du phytoplancton dans la strate 0-10 m à Saint-Victor du 17 mai 1990 au 20 juillet 1991.

Fig. 4. - Changes in the counting of algal cells $\left(10^{6}\right.$ cells per liter) of the phytoplankton in the 0-10 $\mathrm{m}$ layer at the site Saint-Victor between 17-05-90 and 20-07-91.

nos mesures indiquent une teneur moyenne très voisine : $23 \mathrm{mg} \cdot \mathrm{m}^{-3}$.

Les fleurs d'eau ne sont pas dopées par les remontées de phosphore du sédiment, d'une part parce que la stratification thermique est nette et isole rapidement épi et hypolimnion et d'autre part parce que les conditions d'anoxie permettant le relargage n'étant réalisées qu'en fin d'été, soit bien après le développement maximum des cyanobactéries. En fait, la teneur en phosphates de l'épilimnion semble largement suffisante pour entrainer ces proliférations.

\subsection{Le phytoplancton}

Globalement, l'évolution du peuplement phytoplanctonique est similaire aux trois points étudiés. Deux périodes distinctes apparaissent (fig. 4) :

- l'une estivale (du 6 juin au 19 octobre pour cette étude) où le phytoplancton est dominé par des Cyanobactéries, principalement par Microcystis aeruginosa, accompagnée de Anabaena flos-aquae et de Aphanizomenon flos-aquae, espèces capables de fixer l'azote atmosphérique;

- l'autre hivernale (du 27 novembre au 15 mars) où le peuplement est 

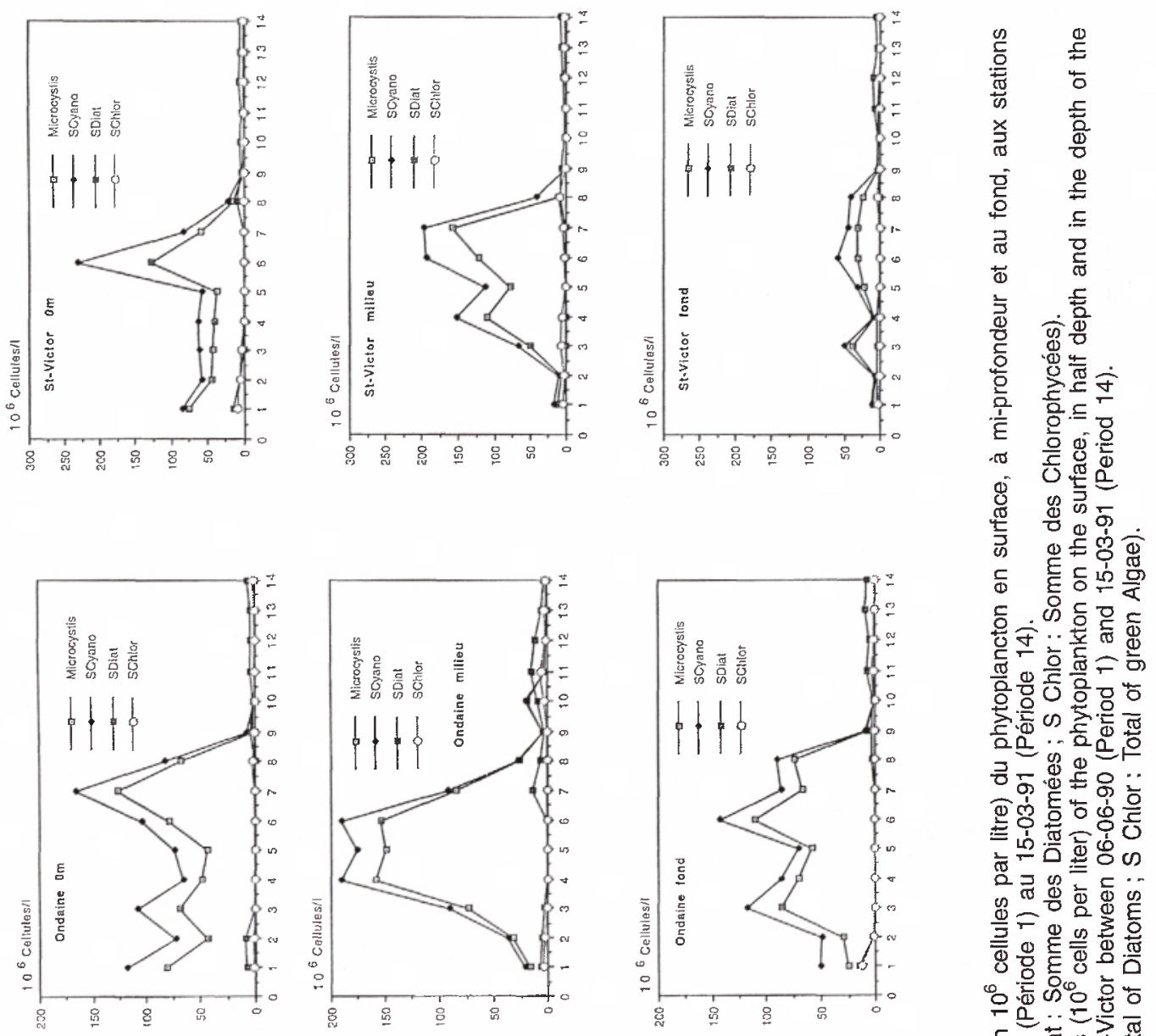

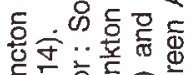

뜨의

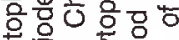

敖的就要

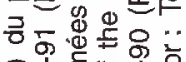

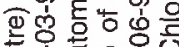

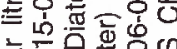

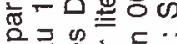

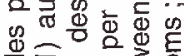
竞 $=0$ $\overline{\mathrm{d}}$ 石

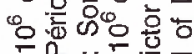

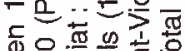

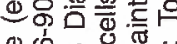
of

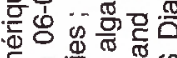

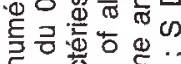

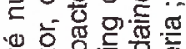

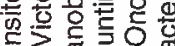

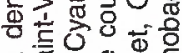

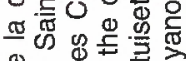
8 ᄃ 0 o

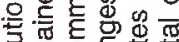

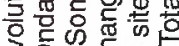

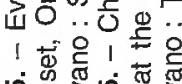
ம.

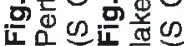


116
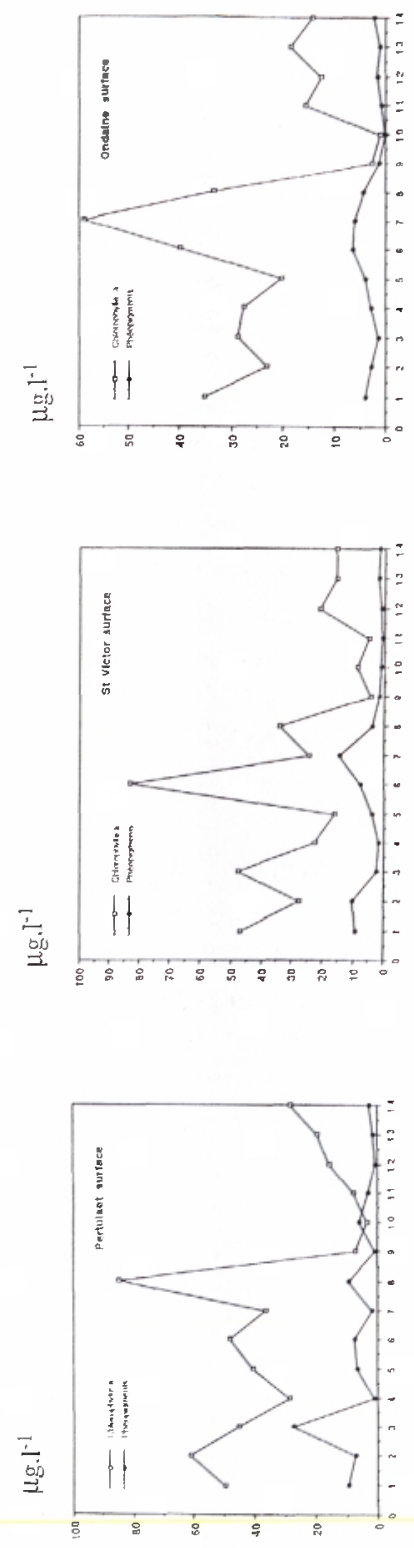

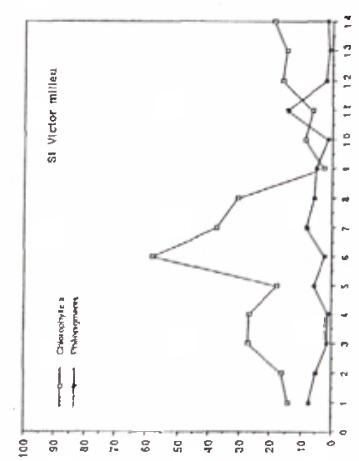

J.L. Berthon et al.
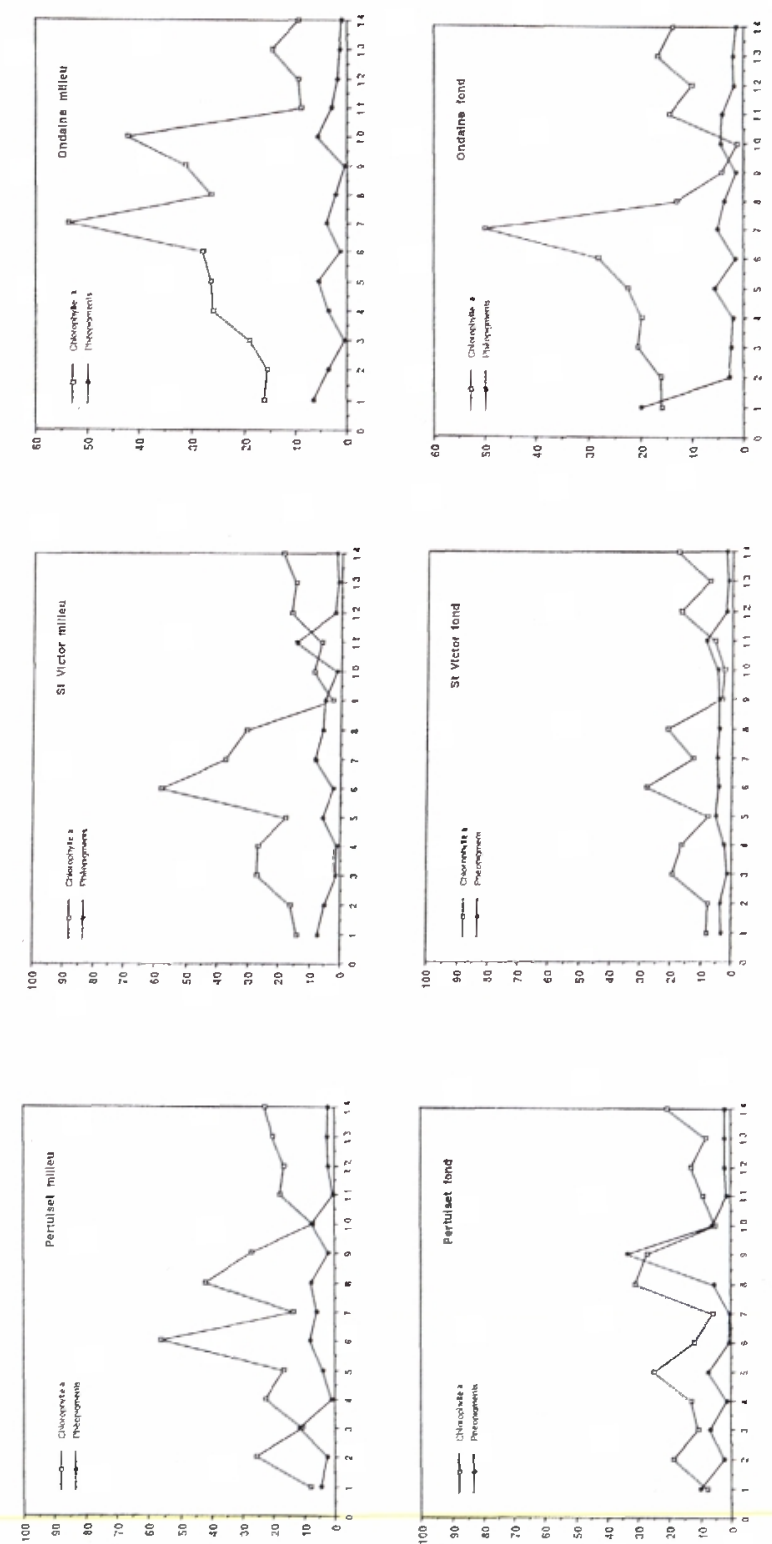

뜽

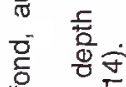

기응

屯. $\subseteq$ के

Э

힘

둥이

은

(i)

$\infty$.

बid

转

का की

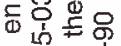

क口 5 它

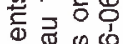

है-

흥

용

든 응

뒁ㅇㅇㅇ

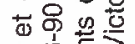

ه

产官. 동

층긍 융

I 은

然

일

亏怘

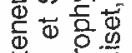

는 글

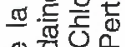

음

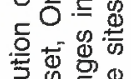

号号宁

西它

๑

온

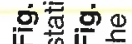


composé de diatomées - Asterionella formosa (espèce ubiquiste) Tabellaria fenestrata, Melosira granulata et Fragilaria crotonensis (typiques des lacs eutrophes) - et de Chlorophycées appartenant aux genres Chlamydomonas, Oocystis, Sphaerocystis et Scenedesmus.

Les pics de diatomées suivent toujours les augmentations de débits du fleuve. Celles-ci accentuent les mouvements de la masse d'eau et alimentent le lac en silice.

Qu'elle soit mesurée ou calculée, l'évolution spatiotemporelle de la biomasse ne suit qu'imparfaitement la densité numérique cellulaire (fig. 5 et 6). Cette discordance est fréquemment observée lorsque les Cyanobactéries dominent le peuplement. En effet, l'échantillonnage des colonies de grande taille de Microcystis est assez aléatoire, puis le dénombrement des cellules dans les colonies n'est qu'approximatif; les biovolumes cellulaires des algues sont très supérieurs à ceux des Cyanobactéries; la teneur en chlorophylle a par unité de volume cellulaire varie considérablement selon les espèces et selon l'état physiologique des cellules (Strikland, 1960; Aleya et al., 1988), enfin, la pesée directe du matériel récolté ajoute à la biomasse, des particules minérales et la nécromasse planctonique. Les résultats obtenus permettent cependant de comprendre la dynamique du peuplement phytoplanctonique en chacun des trois points.

\section{a) Pertuiset (station 3)}

Dès la fin du printemps et durant l'été, le phytoplancton est très largement dominé par Microcystis aeruginosa, avec cependant des fluctuations de la densité numérique. Si l'on considère la teneur en oxygène dissous comme indicatrice de l'intensité de la production primaire, deux phases de croissance de Microcystis aeruginosa apparaissent clairement en été : la première correspond aux trois premiers prélèvements (6-6 au 11-7). La sursaturation en oxygène traduit une photosynthèse intense et donc une population en croissance. La seconde poussée correspond aux 7 et 20-9. Entre les deux, les Cyanobactéries maintiennent des densités moins élevées.

Les biomasses considérables de Cyanobactéries récoltées au Pertuiset ne résultent pas uniquement d'une production locale intense. On a également là une accumulation en surface, de cellules produites plus en amont, dès que le flux d'azote issu du lessivage et véhiculé par la Loire, diminue. Les colonies descendent sous l'action du courant et du vent dominant et s'arrêtent dans la première anse prononcée, au niveau du Pertuiset.

La phase hivernale débute lorsque les Cyanobactéries disparaissent totalement de la masse d'eau (novembre). Mais la disparition de la fleur d'eau est initialisée dès le 15 septembre par la reprise du turbinage des eaux et le marnage qui en résulte. La 
sédimentation des cellules sénescentes apparaît dans l'évolution de la densité numérique cellulaire en profondeur. On observe alors une reprise du développement algal.

\section{b) Ondaine (station 4)}

Dans l'ensemble, l'évolution des populations phytoplanctoniques y est la même que celle décrite au Pertuiset. On observe les deux phases saisonnières. Mais les densités numériques sont toujours peu importantes, de même que les valeurs de biomasse et de chlorophylle a et les eaux sont turbides, chargées de particules inorganiques. L'ACP révèle une dynamique complexe du peuplement algal. Les plus fortes concentrations cellulaires ne sont jamais associées à une sursaturation en oxygène. Ces cellules ne produisent pas ou peu. D'ailleurs leur abondance est maximale dans les échantillons prélevés à mi-profondeur, où là, l'éclairement est insuffisant pour permettre une photosynthèse importante. La plupart des cellules sont sénescentes. Leur teneur en phéopigments est élevée. Ces cellules doivent provenir pour l'essentiel de l'amont. II y a probablement un développement local, mais celui-ci est limité, peut-être par la médiocre qualité des eaux provenant de l'Ondaine, comme le démontrent les mesures de production in situ, réalisées dans le cadre de notre enseignement de Maitrise. Une seule phase de croissance sur place apparait évidente lors du prélèvement du 22-8: on observe simultanément une augmentation de la densité cel- lulaire cyanobactérienne et une sursaturation en oxygène nette. Cette poussée cyanobactérienne à l'Ondaine précède de 13 jours celle enregistrée au Pertuiset. On constate alors que ce développement suit immédiatement une baisse du rapport N/P dans les eaux de l'Ondaine comme de la Loire.

\section{c) Saint-Victor (station 9)}

Comme aux deux autres points, les Cyanobactéries dominent du 6-6 au 19-10, et les Diatomées et les Chlorophycées durant la période automnehiver. Cependant, cette station présente plusieurs caractéristiques originales :

- Les Cyanobactéries y sont beaucoup moins abondantes qu'aux deux stations précédentes, hormis le 22-8. Mais il s'agit là de cellules sénescentes, dérivant de la zone du nouveau port de Saint-Victor, situé à l'embouchure du Lizeron.

- A la profondeur -15 $\mathrm{m}$, on observe durant tout l'été (du 6-6 au 7-9) une augmentation nette de la densité cellulaire et de la teneur en chlorophylle. II s'agit probablement d'une strate d'accumulation de cellules sur une thermocline peu marquée, où l'activité des décomposeurs est réduite puisque la teneur en oxygène est relativement élevée.

- Enfin, en profondeur la biomasse algale et la concentration en chilorophylle sont beaucoup plus faibles qu'aux stations en amont. On peut penser que dans cette zone aval de la retenue la plus grande partie 
des cellules mortes est décomposée avant d'atteindre le fond, à environ $35 \mathrm{~m}$.

Ainsi, il apparait deux sites favorables au développement des Cyanobactéries dans la retenue de Grangent : le Pertuiset et une vaste zone autour du port de Saint-Victor. Les processus y sont assez comparables avec un décalage dans le temps.

On constate que lorsque les Cyanobactéries apparaissent, le développement algal régresse. S'agit-il là du résultat d'une élimination? Les Cyanobactéries empêchiant les algues de se développer, par exemple du fait de leur toxicité? II est probable que non. En effet, le développement de $M$. a. suit toujours le disparition des algues. II ne la précède jamais. II ne s'agit donc pas d'une compétition dont Microcystis sortirait vainqueur. En fait, la toxicité de Microcystis aeruginosa est alors peu importante, lorsque les colonies sont en croissance (Carmichael, 1981) et ses effets sont faibles sur la croissance des algues (Blachetta, 1992). On pourrait également évoquer la diminution de la transparence de l'épilimnion, du fait de l'abondance des colonies cyanobactériennes et/ou la stratification thermique, défavorable aux diatomées qui sédimentent vite en absence de turbulences. Mais le facteur déterminant apparaît être la baisse du rapport N/P. Chacune des phases de croissance de Microcystis aeruginosa fait suite à un abaissement en dessous de 7 du rapport N/P dans les eaux de la Loire, tandis que la diminution densitaire entre les 23-7 et 22-8 suit au contraire une remontée transitoire de ce rapport. Une telle corrélation est rapportée dans plusieurs travaux (Vallentyne, 1974; Aleya et al., 1994). Bien qu'incapable, comme d'autres Cyanobactéries, de fixer l'azote aérien Microcystis aeruginosa est indifférente à cette variation. Elle la tolère, au contraire des algues qui exigent un rapport massique N/P beaucoup plus élevé, supérieur à 7 (voire très supérieur), pour une croissance optimale (Welch et al., 1978).

\subsection{Le zooplancton}

La composition du zooplancton n'est pas très euristique quant à l'état trophique de la retenue. En effet, la plupart des espèces inventoriées sont assez communemment rencontrées dans les grands lacs (Riemann et Sondergaard, 1986).

De l'étude de la cinétique des populations (Berthon, 1988; Devaux, 1992), on retiendra que:

- Le copépode Eudiaptomus gracilis est absent de la station Pertuiset où la profondeur est insuffisante pour cette espèce qui doit effectuer d'amples migrations verticales journalières pour constituer les réserves énergétiques assurant sa survie hivernale (Berthon, 1988).

- Au contraire, Chydorus sphaericus, forme héléoplanctonique, n'est présent qu'au Pertuiset et à l'Ondaine, porté par les amas de Microcystis aeruginosa.

- Le peuplement rotifère est le même aux trois points, mis à part Brachionus calyciflorus absent à Saint- 
Victor et associé aux deux autres points à Microcystis aeruginosa, qu'il consomme (Gilbert \& Starkweather, 1977).

La biomasse zooplanctonique varie considérablement selon les points: les valeurs les plus élevées sont observées au Pertuiset de juin à août puis à l'Ondaine en septembre et octobre. C'est à Saint-Victor que les biomasses les plus faibles sont mesurées (fig. 7).

L'influence de Microcystis sur le zooplancton apparait clairement lorsqu'on considère l'évolution de son contenu énergétique (noté $\mathrm{CE}$ ). $\mathrm{Ce}$ contenu diminue rapidement lorsque la Cyanobactérie est présente. La diminution est importante au Pertuiset surtout lorsque les cellules de Microcystis entrent en sénescence. A l'Ondaine le contenu énergétique des crustacés est très faible durant toute l'année, traduisant leur très mauvais état physiologique, dû à la médiocre qualité de leur nourriture. La valeur de

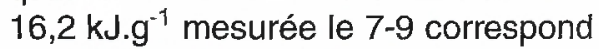
au minimum vital (Berthon, 1988). Le CE est plus élevé à Saint-Victor, où les Cyanobactéries sont moins abondantes et où par ailleurs, la profondeur protège les animaux qui stoppent

Fig. 7. - Evolution de la biomasse et du contenu énergétique du zooplancton aux stations Pertuiset, Ondaine et Saint-Victor, du 06-06-90 (période 1) au 15.03.91 (période 14).

Fig. 7. - Changes in biomass and energetic vaIues of zooplankton at the sites Pertuiset, Ondaine and Saint-Victor between 06-06-90 (period 1) and 15-03-91 (period 14).

$\mathrm{g} \cdot \mathrm{m}^{-3}$

$\mathrm{kJ} . \mathrm{g}-1$
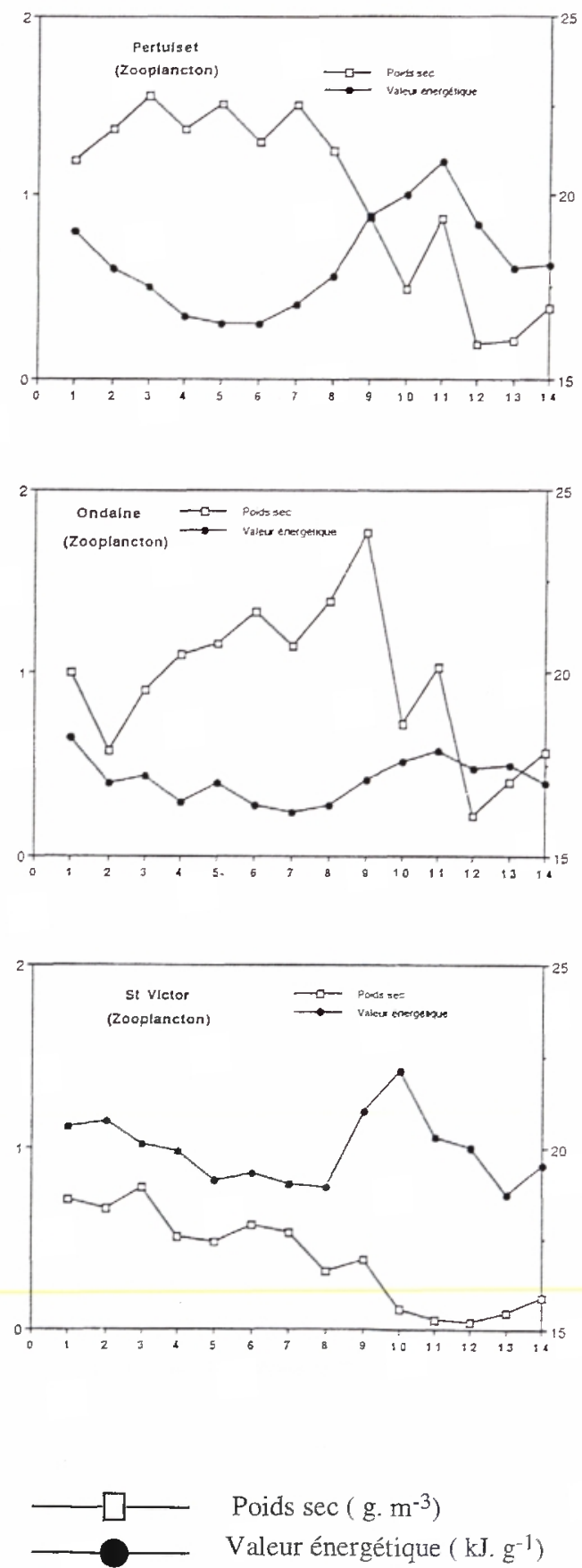

Poids sec ( g. $\left.\mathrm{m}^{-3}\right)$

Valeur énergétique $\left(\mathrm{kJ}, \mathrm{g}^{-1}\right)$ 
leurs migrations verticales, séjournant près du fond, quand l'épilimnion est encombré de cellules mortes de Microcystis.

\section{CONCLUSIONS}

Les résultats obtenus dans cette étude ont confirmé clairement le caractère hyper-eutrophe de la retenue de Grangent. Tous les paramètres retenus par Vollenweider (1968 et 1976) sont ici au dessus des valeurs minimales fixées par cette commission.

Par ailleurs, ils confortent également l'avis de nombreux auteurs quant au rôle prépondérant des petites cellules phytoplanctoniques dans les phénomènes d'extinction de la lumière (Lorenzen, 1980; Paerl et al., 1985; Aleya et Dévaux, 1989): à Grangent les eaux sont très turbides et la profondeur de disparition du disque de Secchi est très faible en été (jamais supérieure à $1,2 \mathrm{~m}$ en été).

Aux stations de mesures du Pertuiset et de l'Ondaine, le plan d'eau n'est stratifié qu'en été, c'est-à-dire lorsque les tributaires sont à l'étiage, tandis qu'à Saint-Victor, la période de stratification de la colonne d'eau s'étend de Mars à Décembre en 19901991, années de faible hydrologie. Ceci indique un caractère pélagique lié à la profondeur. Autrement dit, la zone aval fonctionne comme un écosystème lac, au contraire de la région amont qui est plus proche d'un fleuve élargi. Ainsi, dans la zone aval, autour de Saint-Victor, l'évolution de la composition chimique des eaux est plus fortement influencée par la communauté phytoplanctonique que par les apports exogènes ou en provenance de la zone amont, apports qui expliquent en totalité l'évolution de la région amont de la retenue.

La succession des populations phytoplanctoniques est largement conditionnée par les paramètres physico-chimiques; il en est ainsi dans de nombreux lacs et retenues (Harris, 1980; Kimmel \& Groeger, 1984 ; Aleya \& Dévaux, 1992; Dévaux \& Aleya, 1993). Cette interdépendance s'accentue lorsque le peuplement est dominé par des Cyanobactéries (Reynolds, 1980). La plus grande stabilité de la colonne d'eau, en période estivale, dans la région aval de la retenue et donc le moindre renouvellement des éléments nutritifs disponibles, expliquent pourquoi le développement de Microcystis aeruginosa débute certaines années plus tôt à Saint-Victor qu'à l'amont (et le plus souvent en même temps). C'est le cas en 1990 pour la seconde phase de prolifération, qui débute le 22-8 à Saint-Victor et le 7-9 au Pertuiset : Microcystis aeruginosa semble très sensible aux variations rapides des conditions de vie, même lorsque celles-ci sont faibles (nous avons observé en juin 1991, qu'un simple orage, en déstratifiant très peu de temps le plan d'eau, peut stopper la prolifération cyanobactérienne et provoquer un redéveloppement fugace des algues: fig. 5).

Dans ces conditions, la fleur d'eau de Microcystis aeruginosa qui envahit en quelques jours la retenue de Grangent ne peut s'expliquer par la seule 
dérive de la biomasse produite dans la zone du Pertuiset. En fait il ressort de ce travail qu'elle est le résultat de l'activité de deux foyers de prolifération: le secteur Aurec-Pertuiset et l'estuaire du Lizeron, vers le nouveau port de Saint-Victor. Les apports de la Loire ne sont donc pas les seuls à incriminer.

Si les blooms de Cyanobactéries sont incontestablement liés aux apports excessifs en orthophosphates, ils le sont plus encore à la baisse des apports d'azote. La diminution du rapport N/P ne favorise pas particulièrement les Cyanobactéries, mais en revanche, elle nuit considérablement aux algues qui périclitent aussitôt. Le phénomène de fleurs d'eau de Microcystis aeruginosa dans Grangent apparaît donc sous un jour nouveau. II ne s'agit pas de la conséquence d'une compétition entre des algues et des Cyanobactéries, mais d'une alternance entre ces deux groupes: les Cyanobactéries ne se développent que lorsque les algues se retirent, du fait de conditions de vie devenues défavorables (azote limitant, phosphore suffisant). II en est de même mais en sens inverse, en septembre lorsque le rapport N/P augmente en même temps que la colonne d'eau est brassée. Microcystis, qui n'aime pas les mouvements d'eau et l'instabilité, périclite. Elle laisse la place aux algues qui se développent aussitôt.

En été, la prolifération de M.a. n'est pas limitée par la consommation par le zooplancton herbivore qui ne l'accepte pas comme nourriture (Lampert, 1982; Fulton \& Jones, 1991), voire qui peut éviter son contact, comme le montrent la mise en place de mécanismes d'inhibition des migrations dès que les colonies de M.a. sont sénescentes (Berthon et Brousse, 1995). Pour le zooplancton, la présence des Cyanobactéries équivaut à une absence de nourriture, ce qui se traduit par une baisse de la valeur énergétique des individus, puis par leur mort. En effet, de nombreux organismes ne parviennent pas à assurer leur ration alimentaire de survie par le seul broutage des algues accompagnatrices de M.a. Leur contenu énergétique diminue donc rapidement, dans la mesure où ces organismes consomment petit à petit leurs réserves pour compenser leur jeûne partiel. Ceci conduit inévitablement à un dysfonctionnement de l'ensemble du réseau trophique.

Ainsi, du fait du développement de Cyanobactéries, la lutte contre l'eutrophisation et le dysfonctionnement de la retenue de Grangent ne passe pas uniquement par la diminution des apports largement excessifs en orthophosphates, traitement classiquement utilisé pour résoudre ce problème. $\mathrm{Si}$ l'on souhaite à la fois, réduire la biomasse phytoplanctonique et éliminer les Cyanobactéries, il faut diminuer les apports globaux en nutriments, tout en entretenant les conditions favorables aux Chlorophycées. Donc baisser les apports en phosphore, mais également contrôler ceux d'azote, pour stabiliser le rapport $N / P$ dans une gamme toujours supérieure à 11 .

A la fin de cette étude, il a donc été suggéré de réduire de façon im- 
portante les apports externes en phosphore, par exemple jusqu'à obtenir une teneur moyenne en chlorophylle voisine de $10 \mathrm{mg} \cdot \mathrm{m}^{3}$ (ce qui place encore cette retenue dans la catégorie eutrophe). Ceci revenait, dans les conditions observées en 1990 et 1991, à diviser par 4 environ les apports par les tributaires. Cet objectif était parfaitemment possible à atteindre, compte-tenu du caractère ponctuels des principales sources de phosphore et des systèmes des déphosphatations disponibles.

Une partie de ce travail a été entrepris sous l'égide de l'Agence de l'Eau Loire-Bretagne: déphosphatation des eaux usées des Villes du Puy-en-Velay et d'Aurec par exemple. Depuis 1995, nous avons observé une modification de la communauté phytoplanctonique, caractérisée par :

- un moindre développement des Cyanobactéries, tant en biomasse qu'en durée;

- le maintien de Chlorophycées dans l'épilimnion durant tout l'été.

Autrement dit, nous retrouvons une situation proche de celle qui était décrite avant 1980 (Salençon, 1996). Cette évolution, tout en tenant compte d'une hydraulique différente, pourrait indiquer que l'amélioration est en bonne voie.

\section{RÉFÉRENCES BIBLIOGRAPHIQUES}

Aleya L., Devaux J., El Magouri H., Marvalin O. \& Amblard C., 1988. Usefulness of simultaneous use of several methods for the estimation of phyto- planktonic biomass. Europ. J. Protistol. 23 : 334-342.

Aleya L. \& Devaux J., 1989. Intérêts et signification écophysiologique de l'estimation de la biomasse et de l'activité photosynthétique de diverses fractions de taille phytoplanctoniques en milieu lacustre eutrophe. Rev. Sci. Eau. 2: 253-272.

Aleya L. \& Devaux J., 1992. The concept of seasonal succession theory applied to phytoplankton through the coupling use of diversity index and rank-frequencies diagrams in a eutrophic ecosystem. Internationale Rev. Gesamten. Hydrobiol. 77 : 579-591.

Aleya L., Desmolles F., Michard M., Bonnet M.P. \& Dévaux J., 1995. The deterministic factors of the Microcystis aeruginosa blooms over a biyearly survey in the hyper eutrophic reservoir of of Villerest (Roanne, France). Arch. Hydrobiol., Suppl., 99 : 489-515.

Berthon J.L., 1988. Contenu énergétique, migrations et stratégies du zooplancton lacustre (cas de quelques populations de trois lacs tempérés). Th. Doc. Etat, Univ. de Saint-Etienne. 313 p.

Berthon J.L. et Brousse S., 1995. Modification of migratory behavior of Crustacea in the presence of a bloom of Microcystis aeruginosa (Cyanobacteria). Hydrobiologia, 300/301: 185-193.

Blachetta K., 1992. Effects of Microcystis aeruginosa (Cyanobacteria) on the growth of other phytoplankton species, isolated from an eutrophic lake, Lake Villerest (France). DEA, Univ. Blaise Pascal, Clermont II, $50 \mathrm{p}$.

Brody S., 1945. Bioenergetics and growth. Reinjold, New-York, $1023 \mathrm{p}$.

Carmichael W.W., 1981. The water environment: algal toxins and health. Plenum Press NY, $491 \mathrm{p}$.

Doledec S. \& Chessel D., 1992. ADE Software. Multivariable Analyses and Graphical Display for Environmental Data. Version 3.3 User's Manual. Ecologie 
des eaux douces et des Grands Fleuves. URA CNRS 1451.

Devaux J., 1992. Etude de la retenue de Grangent en 1990. Rapport final, $131 \mathrm{p}$.

Devaux J. \& Aleya L., 1993. A new approch to analyse the phytoplankton community structure. Arch. Hydrobiol. 128 : 287-308.

Feuillade J., 1992. Les toxines des cyanobactéries: revue de synthèse. Rev. Sc. de l'Eau, 5 : 489-508.

Frontier S., 1973. Etude statistique de la dispersion du zooplancton. J. Exp. Mar. Ecol., 12 : 229-262.

Gaillard J., 1981. A predictive model for water quality in reservoirs and its application to selective withdrawal. $\mathrm{Ph}$. D. Dissertation. Colorado State Univ., Fort Collins, $232 \mathrm{p}$.

Garçon V., 1981. Modélisation numérique d'un écosystème aquatique: application au reservoir de Grangent sur la Loire. Th. Doc. $3^{e}$ cycle, Univ. de Paris VII, $230 \mathrm{p}$.

Golterman H.L., Clymo R.S. et Ohnstad M.A.M., 1978. Methods for physical andchemical analyses of freshwaters. IBP $n^{\circ} 8,2 d$ éd. Blackwell Scientific Publication, Oxford, $213 \mathrm{p}$.

Gilbert J.J. \& Starkweather P.L., 1977. Feeding on the rotifer Brachionus calyciflorus. I. Regulatory mechanisms. Oecologia, 28 : 125-131.

Gower J.C.A., 1971. A general coefficient of similarity and some of its properties. Biometrics, 27 : 857-871.

Juday C., 1940. The annual energy budget of an inland lake. Ecology, 21: 438-450.

Lampert W., 1982. Further studies on the inhibitory effects oh the toxic bluegreen algae Microcystis aeruginosa on the filtering rate of zooplankton. Arch. Hydrobiol., 95 : 207-220.
Legendre L. \& Watt W.D., 1971-1972. On rapid technique for enumeration. Ann. Inst. Oceanogr., Paris, 58 : 173-177.

Lohman H., 1908. Untersuchungen zur Feststellung des Vollständigen Gehaltes des Meeres an Plankton. Wiss. Meeresunters, Abt. Kiel N.F. 10 : 132170.

Lorenzen C.J., 1967. Determination of chlorophyll and pheopigments : spectrophotometric equations. Limnol. Oceanogr, 12, 2 : 343-346.

Paerl H.W., Bland P.T., Bowles N.D. \& Haibach M.E., 1985. Adaptation to highintensity, low wavelength among surface blooms of the Cyanobacterium Microcystis aeruginosa. Appl. Environ. Microbiol. 49 : 1046-1052.

Reynolds C.S., 1984. Artificial introduction of surface blooms of Cyanobacteria. Verh. Int. Ver. Limnol. 22 : 638-643.

Riemann B. \& Sondergaard M., 1986. Carbon dynamics in eutrophic temperate lakes. Elsevier Ed. 284 p.

Rohlich G.A. et Uttormark P.D., 1972. Wasterwater treatment and eutrophisation. Am. Soc. Limnol. Oceanogr. Spe. Symp. 1 : 231-245.

Salençon M.J., 1995. Evolution de l'écosystème de la retenue de Grangent de 1978 à 1992. Rapport EDF DER, HE - 31/95/012, 45 p.

Saunier Eau et Environnement, 1988. Impacts des rejets stéphanois en phosphore sur la qualité de la Loire. Rapport, $39 \mathrm{p}$.

SCOR-UNESCO, 1966. Determination of phytoplancton pigments in sea water. SCOR-UNESCO, $69 \mathrm{p}$.

Strickland J.H., 1960. Measuring the production of marine phytoplankton. Bull. Fish. Res. Board Can. 122, 172 p.

Utermöhl H., 1958. Zur Vervollkommung der quantitative Phytoplankton-Methoik. Mitt. Intern. Ver. Limnol. 9:1-38. 
Vallentyne J.R., 1974. The algal Bowl lakes and Man. Misc. Spec. Publ. 22, Dept Environment, Fish. mar. ser., Ottawa, $196 \mathrm{p}$.

Vollenweider R.A., 1968. The scientific basis of lake eutrophication, with particular reference to phosphorus and nitrogen as eutrophication factors.
Tech. Rep. D.A.S./C.S./68.2O.C. D.E. Paris, $159 \mathrm{p}$.

Vollenweider R.A., 1976. Advances in defining critical levels for phosphorus in lake eutrophication. Mem. Ist. Ital. Idrobiol., 33 : 53-83.

Wetzel R.G., 1983. Limnology, Saunders College, New-York, $2^{e}$ éd., $767 \mathrm{p}$. 